\title{
Chirurgische Infektionen bei Kindern und Jugendlichen
}

\author{
Guido Fitze und Christian Kruppa
}

\section{Inhalt}

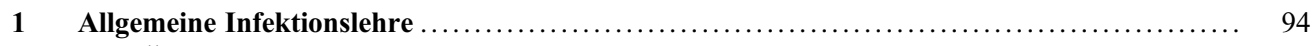

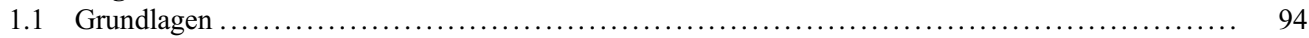

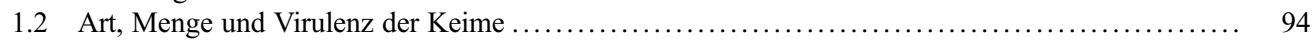

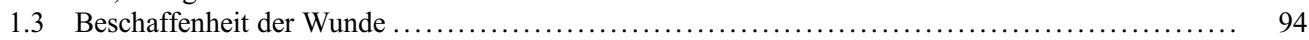

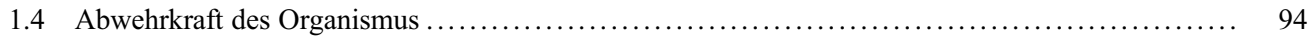

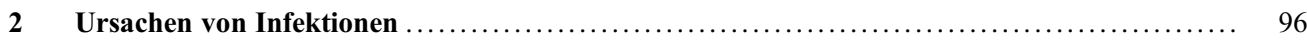

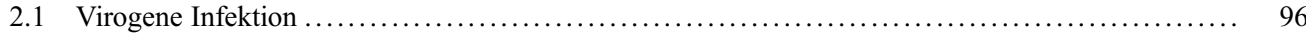

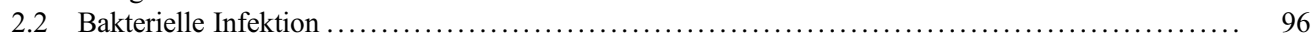

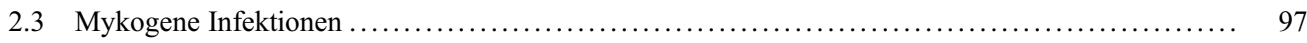

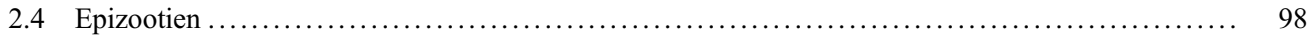

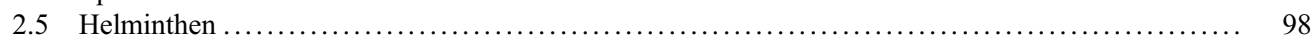

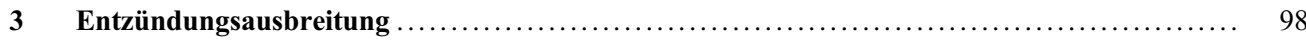

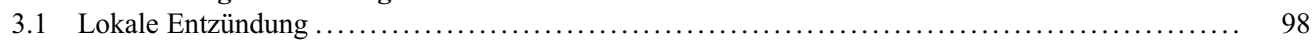

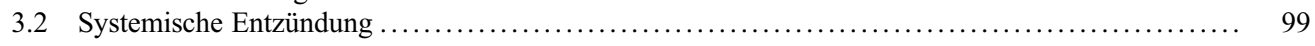

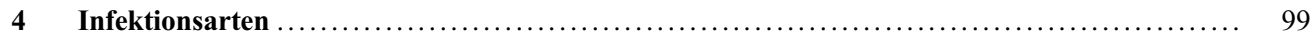

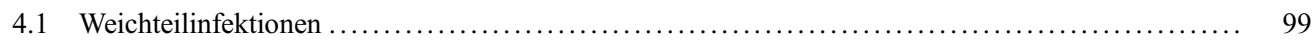

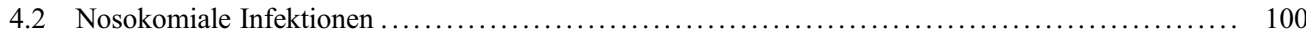

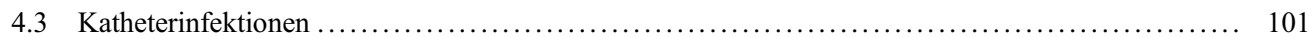

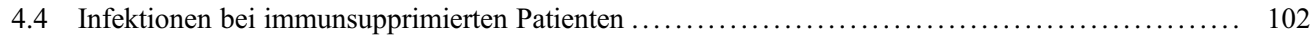

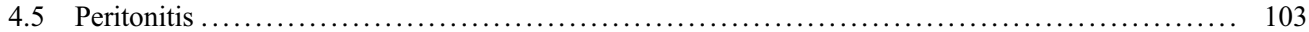

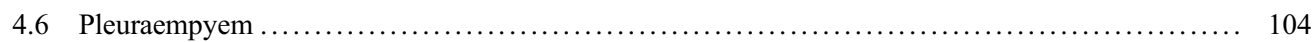

$5 \quad$ Grundsätze der chirurgischen Infektionsbehandlung $\ldots \ldots \ldots \ldots \ldots \ldots \ldots \ldots \ldots \ldots \ldots \ldots \ldots \ldots$

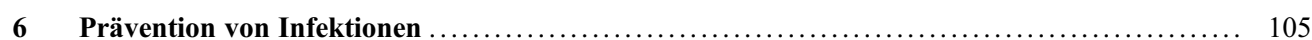

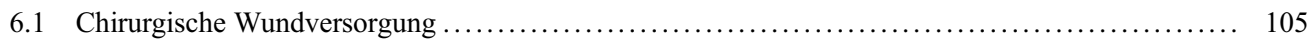

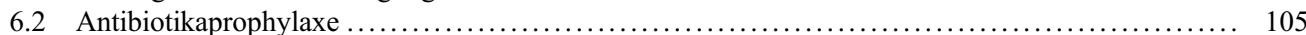

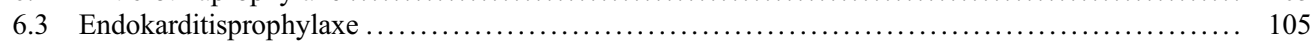

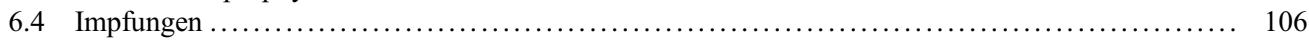

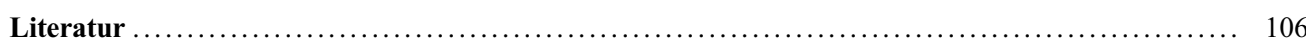

Der menschliche Organismus ist von einer unzähligen Menge von Mikroorganismen umgeben. Dringen diese

G. Fitze $(\bowtie) \cdot$ C. Kruppa

Klinik und Poliklinik für Kinderchirurgie, Universitätsklinikum Carl

Gustav Carus, Dresden, Deutschland

E-Mail: Guido.Fitze@uniklinikum-dresden.de;

christian.kruppa@uniklinikum-dresden.de
Krankheitserreger in uns ein, dann laufen Wechselbeziehungen zwischen dem Eindringling und dem Wirtsorganismus ab. Krankheit resultiert, wenn die Mikroorganismen aufgrund ihrer verschiedenen Determinanten der Pathogenität und Virulenz den Wettlauf mit der Infektabwehr des Makroorganismus gewonnen haben. Mit diesem Problem hatten sich Chirurgen zu allen Zeiten auseinanderzusetzen und es hat bis heute nicht an Aktualität verloren. 
1 Allgemeine Infektionslehre

\subsection{Grundlagen}

Chirurgisch relevante Infektionen werden überwiegend durch Bakterien hervorgerufen. Als Krankheitserreger müssen aber auch Viren, Pilze, Protozoen sowie Würmer in Betracht gezogen werden. Davon abzugrenzen sind entzündliche Reaktionen, die durch Toxine anderer Organismen wie Insekten oder Schlangen hervorgerufen werden. Die Besiedlung einer Wunde mit pathogenen Keimen führt nicht zwangsläufig zu einer Infektion. Dieser Vorgang wird allgemein als Kolonisation bezeichnet und erfolgt meist als Schmierinfektion, selten aerogen oder hämatogen. Entscheidend für den Übergang in eine krankheitsrelevante Wundinfektion sind:

- Art, Menge und Virulenz der pathogenen Keime,

- Beschaffenheit der Wunde,

- Abwehrkraft des Organismus.

Die Zeitspanne zwischen der stattfindenden Kolonisation und der klinischen Manifestation einer Wundinfektion wird als Inkubationszeit bezeichnet. Ihre Länge hängt wiederum von Art, Virulenz und Zahl der Erreger, aber auch von der Beschaffenheit der Wunde sowie der Abwehrsituation des Wirtsorganismus ab.

Häufig imponieren Infektionen als Mischinfektionen, bei der Keime unterschiedlicher Erregergruppen beteiligt sind. So können in einer Wunde aerob wachsende Bakterien durch ihren Verbrauch von Sauerstoff die Vermehrung anaerober Bakterien fördern und somit parallel die Infektion unterhalten. Sind verschiedene Bakterien im infizierten Gewebe nachweisbar, die allerdings einer Erregergruppe angehören, so spricht man von einer Polyinfektion. Dagegen liegt eine Monoinfektion vor, wenn nur ein Erreger nachgewiesen wird.

Das klinische Erscheinungsbild einer lokalen Infektion wird in den meisten Fällen durch die klassischen Kardinalsymptome einer Entzündung charakterisiert: Rötung (rubor), Schwellung (tumor), Überwärmung (calor), Schmerz (dolor) sowie Funktionsstörung (functio laesa). Während bei einer akuten bakteriellen Infektion diese Symptome meist ausgeprägt sind, können sie bei chronischen Infektionen oder aber auch bei speziellen Infektionen (bakteriell-toxische Infektion wie Tetanus oder Pilzinfektionen) weniger eindrucksvoll auftreten oder aber sogar ganz fehlen.

\subsection{Art, Menge und Virulenz der Keime}

Art und Menge der pathogenen Keime sind entscheidend für das Auftreten von bestimmten Infektionen des Körpers. So wurde in Experimenten gezeigt, dass eine Keimzahl von mindestens 5 Mio. Keimen von Staphylococcus aureus auf einer oberflächlichen Wunde zu einer manifesten Infektion führen kann. Eine andere Betrachtungsweise setzt eine bestimmte Keimlast für ein Gewebe voraus, um eine Infektion hervorrufen zu können. Dabei wird für bakterielle Infektionen allgemein eine Keimzahl von 100.000 Bakterien pro Gramm Gewebe als Schwellenwert angesehen. Als Beispiel sei hier das Waterhouse-Friderichsen-Syndrom genannt. Hier führt die Virulenz der verursachenden Keime (Meningokokken) innerhalb von wenigen Stunden zu einer Überschwemmung des Organismus mit diesen pathogenen Keimen.

Die Virulenz der pathogenen Keime kann sich durch die Änderung der Umwelt wesentlich verstärken. So kann das normalerweise in der Ostsee ubiquitär als nichtpathogener Keim vorkommende Bakterium Vibrio vulnificus durch Erhöhung der Wassertemperatur $>20^{\circ} \mathrm{C}$ seine Virulenz erhöhen und somit für den Menschen auch gefährlich werden. Vermehrte Wundinfektionen an der Haut konnten durch diese Bakterien in heißen Sommern nachgewiesen werden.

\subsection{Beschaffenheit der Wunde}

Neben dem Verschmutzungsgrad einer Wunde und den vorhandenen Gewebetrümmern infolge einer traumatischen Verletzung spielt auch die lokale Durchblutung der Wunde eine wesentliche Rolle. Das heißt, Risswunden sind für Infektionen empfänglicher als Wunden mit glatten Wundrändern. In situ verbleibende Nekrosen und Gewebetrümmer begünstigen das Auftreten von Infektionen. Auch die Lokalisation der Wunden spielt eine wesentliche Rolle. So heilen Wunden in besser durchbluteten Bereichen wie Gesicht oder Kopfhaut außerordentlich gut, dagegen an der weniger perfundierten Fußsohle wesentlich schlechter. Außerdem steigt die Keimzahl, die zur Infektion einer Wunde führen kann, um mehrere Zehnerpotenzen, wenn sich in der Wunde Fremdkörper befinden. Dies gilt nicht nur für solche, die durch eine mögliche Verletzung von außen eingetragen wurden, sondern auch für solche, die durch chirurgische Maßnahmen in die Wunde gelangt sind (Nahtmaterial, Drainagen oder Katheter).

\subsection{Abwehrkraft des Organismus}

Bei immunsupprimierten Patienten ist durch die entsprechend durchgeführte Therapie die allgemeine Abwehrlage des Patienten verschlechtert. Auch bekannte Nebenerkrankungen wie Diabetes mellitus führt zu vermehrten Wundinfektionen bzw. zu chronischen Wundheilungsstörungen. Ebenso verhindert eine intakte anatomische Barriere, d. h. eine intakte Haut bzw. Schleimhaut, das Eindringen von pathogenen Keimen. So vermindern der niedrigere $\mathrm{pH}-$ Wert 
(ca. 5,5) auf unserer Haut sowie die um ca. $5{ }^{\circ} \mathrm{C}$ niedrigere Temperatur auf unserer Körperoberfläche im Vergleich zur physiologischen Körperkerntemperatur das Wachstum der Bakterien.

Eine wichtige Voraussetzung für die Vermeidung von Infektionen stellt die im menschlichen Organismus natürlich existierende Homöostase der saprophytisch kolonisierenden Keime dar. Wird diese durch meist medizinisches Eingreifen - beispielsweise durch eine Antibiotikagabe verändert, kann dies wiederum eine Infektion induzieren. Als Beispiel sei die nahezu ausnahmslos im Zusammenhang mit einer Antibiotikatherapie auftretende Infektion mit toxinbildendem Clostridium difficile genannt, die in bis zu $20 \%$ aller antibiotikaassoziierten Diarrhoeen und in $90 \%$ aller antibiotikaassoziierten pseudomembranösen Kolitiden die Ursache darstellt. Nahezu jedes in Verwendung befindliche Antibiotikum kann diese Erkrankung auslösen. Nur in Einzelfällen können diese Bakterien auch bei nicht antibiotisch vorbehandelten Kindern eine Kolitis auslösen, jedoch haben diese Patienten in aller Regel eine andere Grunderkrankung wie den Morbus Hirschsprung. Problematisch ist das weite klinische Spektrum der durch Clostridium difficile ausgelösten Erkrankungen, das von einer milden, selbstlimitierenden Diarrhoe über eine behandlungsbedürftige Enterokolitis bis hin zum schweren toxischen Megakolon mit Darmperforation reicht, wobei letztere in 35-50 \% der Fälle wegen eines septischen Multiorganversagens letal verlaufen kann (Mc Laughlin et al. 2014).

Eine intakte humorale und eine zelluläre Abwehr sind unbedingt zur Infektionsprophylaxe erforderlich. So gilt allgemein eine Verminderung der Granulozyten unter eine absolute Zahl von $0,5 \mathrm{Mpt} / \mathrm{ml}$ als infektionsgefährdend und bedingt eine systemische Antibiose als Infektionsprophylaxe. Da die Milz im Wesentlichen für die primäre Immunantwort verantwortlich ist, kann eine Infektion bei asplenischem Zustand zu einer schweren systemischen Infektion (OPSISyndrom) führen (Reid 1994) ( $\triangleright$ Kap. 7, „Splenektomie bei Kindern und Jugendlichen mit hämatologischen Erkrankungen"). Die häufigsten Erreger für eine solche Sepsis sind mit ca. $60 \%$ Streptococcus pneumoniae und mit etwa $30 \%$ Haemophilus influenzae und Neisseria meningitidis. Dagegen spielen Staphylococcus aureus, Escherichia coli und andere gramnegative Erreger eher eine untergeordnete Rolle. Erleiden asplenische Kinder eine Malaria, verläuft diese wesentlich schwerer. Nach Hundebissverletzungen wurden bei diesen schwere Sepsisfälle, ausgelöst durch Capnocytophaga canimorsus, beobachtet. Deswegen ist dem Milzerhalt im Kindesalter uneingeschränkte Beachtung zu schenken. Nach erlittenem Trauma sollten alle konservativen und milzerhaltenden operativen Maßnahmen einschließlich der Teilresektion ausgeschöpft werden. Nach heutiger Ansicht reichen etwa $30 \%$ erhaltenen Milzgewebes aus, um eine normale Milzfunktion zu ermöglichen.
Bei den Immundefekten unterscheiden wir einen primären von einem sekundären Defekt. Erstmals beschrieb Bruton im Jahre 1952 eine Patientin mit einem Antikörpermangel. Laut aktueller Version der Europäischen Immundefektdatenbank (Knerr et al. 2008) sind 209 Immundefekte bekannt, wobei die Antikörpermangelerkrankungen mit einem Anteil von ca. $69 \%$ in Deutschland die größte Gruppe einnehmen, gefolgt von Phagozyten/Wund-T-Zelldefekten. Die betroffenen Patienten sind anfällig für eine Vielfalt rezidivierender Infektionen. Die Ursachen für die primären Immundefekte sind angeborener Natur, d. h. primär genetisch bedingt, die sekundären Immundefekte werden erworben durch andere Krankheiten, Medikamente und auch Umwelteinflüsse. Für erworbene Immundefekte seien als Beispiele die malignen Lymphome, die chronische lymphatische Leukämie, die bakterielle Sepsis bei immunsupprimierten Patienten, die HIV-Infektion, aber auch Hypogammaglobulinämien beim nephrotischen Syndrom oder schwere Verbrennungen genannt.

Im Gegensatz zum Erwachsenen ist das Immunsystem bei Kindern unreif. Sie weisen auch keinen großen Pool von Plasmazellen auf. Aus dieser damit verbundenen relativen B-Zell-Defizienz, wobei das Spektrum der molekularen B-

Zell-Defekte groß ist, resultiert ein erhöhtes bakterielles Infektionsrisiko.

Bei kombinierten Immundefekten (SCID = ,severe combined immunodefiency“ und CVID $=$,,common variable immunodeficiency") ist auch im Kindesalter ein Antikörpermangel vorhanden. Einige Patienten verfügen nur über Vorläufer und keine reifen B-Zellen. Die Ursache dafür liegt in Mutationen verschiedener Gene, die in der Entwicklung und Differenzierung der B-Zellen von Bedeutung sind.

Klassische pathogene Erreger, die infektassoziierte Immundefekte auslösen sowie das Immunsystem modulieren können, sind häufig Viren, z. B. Masernvirus, Zytomegalie-Virus, Ebstein-Barr-Virus, Rötelnvirus und natürlich das HI-Virus. Bei bakteriellen Infektionen stehen die Borrelien, Pneumokokken und Streptokokken im Vordergrund, seltener dagegen die Tuberkulose- und die Lepra-Erreger. Auch Parasiten wie Plasmodien (Malaria), Leishmanien und Trypanosomen, d. h. sog. vektorassoziierte Erkrankungen, die im Rahmen der globalen Erwärmung zukünftig auch in Europa durchaus auftreten könnten, dürften zu Immundefekten führen.

Bei der HIV-Infektion handelt es sich primär um einen T-Zell-Defekt, aber auch um ein sog. Antikörperparadoxon. In diesem Fall liegt trotz einer Hypergammaglobulinämie ( $\mathrm{IgG}$ und $\operatorname{Ig} \mathrm{A}$ ) eine humorale Immundefizienz vor. Diese Immunstörungen sind charakterisiert durch eine ungenügende Bildung von neutralisierenden Antikörpern gegen HIV und Zytomegalie-Virus, durch ein schlechtes Ansprechen auf Impfungen sowie durch eine gestörte 
De-novo-Immunantwort gegen neue Erreger. Eine Substitution mit IgG muss generell bei einer Serumkonzentration $<200 \mathrm{mg} / \mathrm{dl}$ vorgenommen werden. Dabei sollte ein Plasmaspiegel von 600-800 mg/dl angestrebt werden (Bjorkander et al. 2006).

\section{$2 \quad$ Ursachen von Infektionen}

\subsection{Virogene Infektion}

Wichtige virogene Infektionen sind die vulgären Warzen mit bevorzugtem Sitz an Händen, Füßen und Fußsohlen. Die Spitzenkondylome kommen im intertriginösen Bereich sowie an der Genital- und Analschleimhaut vor. Prädisponierender Faktor ist $u$. a. eine bestehende Oxyuriasis. Auch an die Übertragung durch sexuellen Missbrauch bei Kindern ist zu denken.

\subsection{Bakterielle Infektion}

Eine bakterielle Infektion erfolgt über eine Kontakt- oder Schmierinfektion, wesentlich seltener aerogen oder hämatogen. Je nach Bakterientypen unterscheiden wir pyogene, putride, anaerobe, bakteriell-toxische und spezifische Wundinfektionen. Diese gehen mit einer entsprechenden Eiterbildung mit typischer Farbe, Konsistenz und Geruch einher.

\section{Pyogene Infektion}

Der wichtigste Erreger für eine pyogene Wundinfektion aerober Natur ist Staphylococcus aureus. Der Eiter ist dabei gelblich, geruchlos, aber rahmig. Bei einer Infektion mit Streptococcus pyogenes ist der Eiter dagegen gelblich-grauer Natur und dünnflüssig. Eine Pseudomonas-aeruginosaInfektion weist einen süßlich riechenden, grünlich-blauen, dünnflüssigen Eiter auf. Die Staphylococcus-aureus-Infektion ist häufig lokal begrenzt und führt meist zu einem Abszess. Andere Erreger für typische Wundinfektionen sind Escherichia coli, Enterokokken, Proteus und Klebsiellen.

\section{Putride Wundinfektion}

Putride Wundinfektionen werden häufig verursacht durch Mischinfektionen von anaeroben und aeroben Keimen. Im Gegensatz zur pyogenen Infektion handelt es sich in der Regel um eine fortschreitende phlegmonöse Entzündung. Die wichtigsten Erreger sind Proteus vulgaris, Streptococcus anaerobicus, Streptococcus putridus und Clostridien. Diese führen zu einer serösen Exsudation mit einer übel riechenden fauligen Nekrose, in der Regel zu einer Gangrän oder zu einer phlegmonösen Entzündung. Differenzialdiagnostisch muss in diesem Fall der Gasbrand ausgeschlossen werden.

\section{Anaerobe Wundinfektion}

Die wichtigsten Erreger für eine anaerobe Wundinfektion sind Escherichia coli, Bacteroidis fragilis, anaerobe Kokken und Fusobakterien. Diese führen zu einem fäkulent stinkenden, gelblich-rötlich bis blau-schwarz gefärbten, rahmig-eitrigen Exsudat und werden in der Praxis häufig als sog. stinkende Abszesse bezeichnet. Unbehandelt schreitet die Entzündung fort.

\section{Bakteriell-toxische Wundinfektion}

Die typischen Erreger bakteriell-toxischer Wundinfektionen sind die obligat anaerob lebenden Clostridium tetani und Clostridium perfringens sowie Corynebacterium diphtheriae. Obwohl die Eintrittspforte eine gestörte anatomische Barriere darstellt, führen sie im Gegensatz zu anderen Erregern kaum zu einer lokalen Reaktion. Dafür haben sie aber eine allgemeine toxische Wirkung.

\section{Gasbrand}

Clostridium perfringens ist der Haupterreger für den Gasbrand. Die Infektion wird begünstigt durch eine ausgeprägte Gewebstraumatisierung und Ischämie. In sauerstoffarmen und nekrotischen Wunden vermehren sich die Exotoxin bildenden Bakterien sehr rasch. Die lokale Weichteilinfektion geht mit einer Gasbildung einher. Die Toxinbildung führt zu einer toxischen Kapillarschädigung mit foudroyant verlaufender Gewebenekrose und konsekutiver Toxinämie sowie frühzeitiger Hämolyse. Der Lokalbefund ist charakterisiert durch eine deutliche Schwellung, starken Schmerz, violettschwarze Wundfarbe und fleischwasserfarbenes Wundsekret. Durch die lokale Gasbildung wird bei Palpation das typische Knistern im Wundbereich hervorgerufen. Der Befund zeigt eine schnelle Progredienz. Systemische Symptome sind Schock, Ikterus und akutes Nierenversagen.

Therapeutisch steht die großzügige Freilegung der Wunde mit einer radikalen Abtragung aller Nekrosen im Vordergrund. Ergänzt wird diese chirurgische Maßnahme von einer Antibiotikatherapie (Penicillin und Metronidazol). Außerdem kann eine zeitgleich durchgeführte hyperbare Sauerstofftherapie die Heilung (Smith-Slatas et al. 2006) fördern oder als Ultima ratio auch eine Amputation der betroffenen Extremität in Erwägung gezogen werden.

\section{Tetanus}

Neben dem Gasbrand zählt der Tetanus ebenfalls zur Gruppe der bakteriell-toxischen Wundinfektionen. Er wird durch das ubiquitär vorkommende, Sporen bildende Clostridium tetani hervorgerufen. Voraussetzung für den Übergang der Sporen in die vegetative Form sind anaerobe Verhältnisse. Die Inkubationszeit beträgt bis zu 60 Tage. Durch die Freisetzung der Toxine (Tetanospasmin, Tetanolysil) wird eine fortschreitende Lähmung der quer gestreiften Muskulatur hervorgerufen. Unspezifische Symptome sind Lichtscheu, Opisthotonus, 
Risus sardonicus, Unruhe und eine zunehmende absteigende tonische Muskelstarre. Später treten generalisierte Krampfanfälle, eine Hyperthermie und eine Atemlähmung mit begleitender Aspirationspneumonie auf. Schließlich führt der Verlauf zum Herzstillstand.

Die Therapie basiert auf einer ausgiebigen Wundausschneidung, Gabe von Tetanus-Hyperimmunglobulin, simultanen Impfung, Antibiotikatherapie (Penicillin) und einer Intensivtherapie mit Abdunklung, Hyperalimentation, Sedierung, Muskelrelaxation sowie Beatmung.

Cave: Trotz rechtzeitiger Therapie ist die Letalität bei Tetanus hoch (bis zu $50 \%$, bei Neugeborenen bis $100 \%$ ). Daher ist die prophylaktische aktive Tetanusimmunisierung dringend zu empfehlen.

\section{Spezifische Wundinfektion}

Erreger für typische spezifische Wundinfektionen, die häufig Fisteln bilden und chronisch-rezidivierende Verläufe zeigen, sind atypische Mykobakterien. Diese Infektionen manifestieren sich häufig in den zervikalen Lymphknoten und den angrenzenden Weichteilen (Abb. 1). Hinweis für das Vorliegen einer Mykobakterieninfektion stellt der typische intraoperative Befund einer verkäsenden Nekrose dar. Die Diagnose sollte pathologisch, mikrobiologisch und molekulargenetisch gesichert werden.

Die Lymphknotenpakete sind meist sehr verbacken und lassen die Exstirpation problematisch erscheinen. Trotzdem gilt die Lymphknotenexstirpation aller betroffenen Areale als Therapie der Wahl, da sie in $>90 \%$ der Fälle ohne Antibiose zur Heilung führt. Die alleinige Abszessinzision resultiert häufig in langwierigen Fistelungen, die eine sekundäre
Exstirpation notwendig machen. Wenn dies nicht möglich ist, z. B. bei Befall von Lymphknoten innerer Körperregionen oder der Gefahr, angrenzende anatomische Strukturen zu schädigen, kann eine mindestens 6-monatige konservative, dreifache antimykobakterielle Therapie erfolgreich sein. Weitere Erreger für spezifische Wundinfektionen stellen Mycobacterium leprae und Mycobacterium tuberculosis sowie Actinomyces israelii dar.

\subsection{Mykogene Infektionen}

Bedeutungsvoll sind hier die Candida-Mykosen, der Soor. Er kann bei Säuglingen besonders im Mundbereich vorkommen. In diesem Fall stellt er bei ausgeprägtem Befall eine Kontraindikation zu einer Narkose dar. Aber auch beim Auftreten im Windelbereich (Windeldermatitis) findet sich die Infektion häufig in einem zu operierenden Gebiet, z. B. bei einer Leistenhernie, sodass auch hier eine Kontraindikation zu einem operativen Eingriff besteht. Die lokale Behandlung dieser Candida-Mykose steht dann präoperativ im Vordergrund.

Neben dieser relativ harmlosen Infektion wird dagegen eine Candida-Sepsis im Rahmen einer nosokomialen Infektion auf pädiatrisch-onkologischen und Intensivstationen beobachtet. Sie wird begünstigt durch eine Immunsuppression bzw. durch den Einsatz intensivmedizinischer Technik und breiter Antibiotikatherapie, aber auch bei Vorhandensein zentralvenöser Katheter.

Neben den Kandidosen komplizieren nicht selten auch auftretende Aspergillosen den Verlauf der Grunderkrankung bei diesen Patienten. Diese sind v. a. im Lungenbereich nachweisbar und erfordern neben einer systemischen antimykotischen Therapie oft eine chirurgische Sanierung (Abb. 2).
Abb. 1 Atypische

Mykobakteriose zervikal rechts bei einem 23 Monate alten Knaben im MRT. Im rechten Bild ist die Abszedierung mit Fistelbildung dargestellt
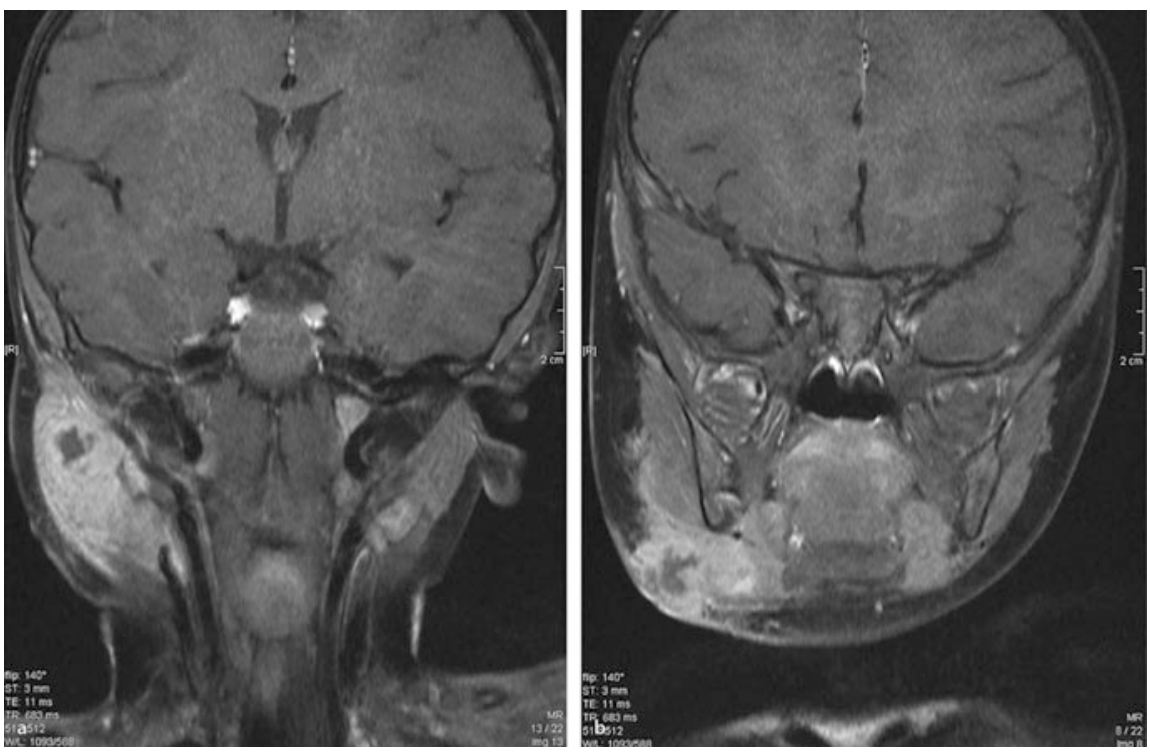

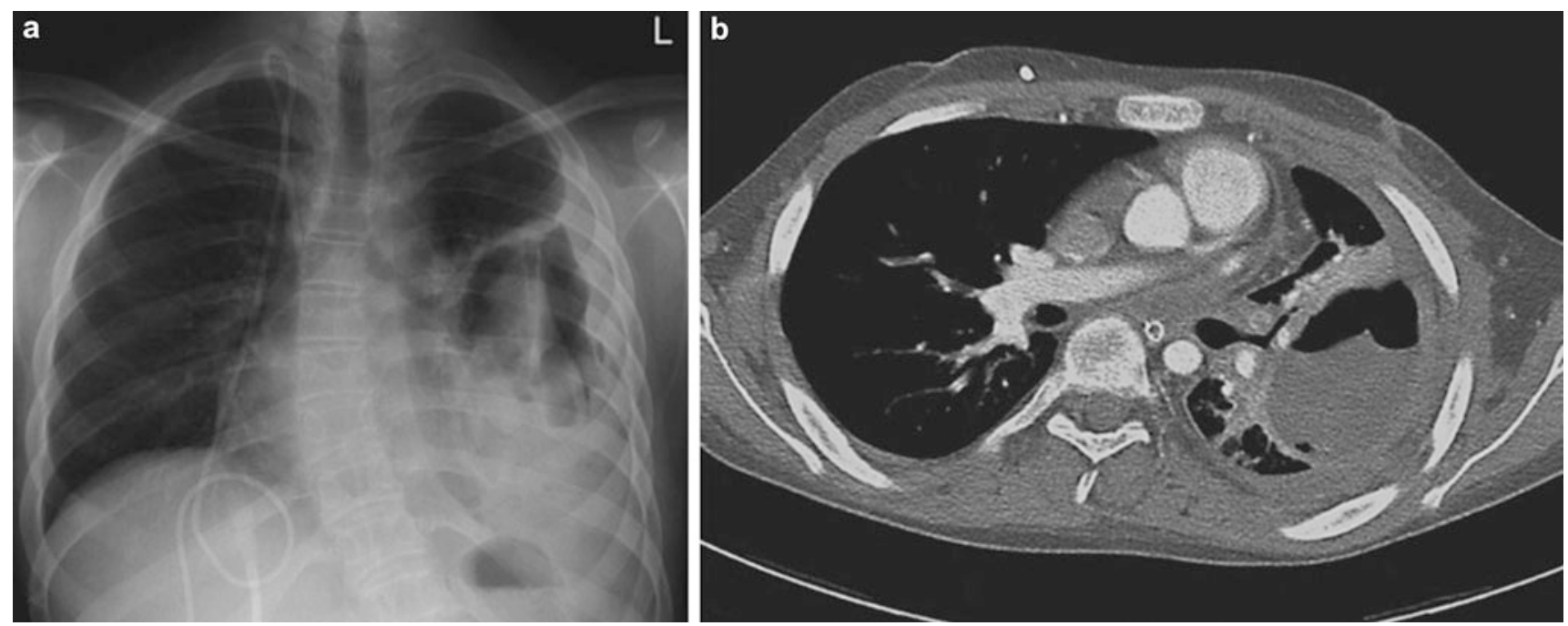

Abb. 2 Aspergillose der linken Lunge beim immunsupprimierten Patienten wegen akuter Lymphoblastenleukämie. a Röntgenübersichtsaufnahme. b Thorax-CT

\subsection{Epizootien}

Als bedeutungsvollste Epizootien seien die Skabies (die Krätze) und der Zeckenbiss durch den Holzbock genannt. Bei Letzterem kommt in Europa die Spezies Ixodes ricinus vor, die durch den Biss das FSME-Virus übertragen kann. Dies ist in den Endemiegebieten (in Deutschland Bayern, Baden-Württemberg, Hessen sowie in einzelnen Gebieten von Thüringen, Sachsen, dem Saarland und Rheinland-Pfalz) epidemiologisch von Bedeutung. Die Durchseuchung der Zecken mit dem Virus beträgt momentan in diesen Gebieten bis zu $1 \%$ und die Infektion mit diesem Virus kann zur Frühsommermeningoenzephalitis (FSME) führen. Zur Prophylaxe dieser viralen Erkrankung steht eine aktive Immunisierung zur Verfügung, die Personen in den Endemiegebieten empfohlen wird, die sich oft in der freien Natur aufhalten.

Neben dem FSME-Virus übertragen die Zecken jedoch einen zweiten Krankheitserreger - das Bakterium Borrelia burgdorferi, den Erreger der Lyme-Borreliose. Primäraffektion für diese Infektion stellt das Erythema chronicum migrans dar. Unbehandelt kann das Bakterium über Blutund Lymphbahnen im Körper disseminieren, verschiedene Organmanifestationen zeigen (Arthritiden, Neuroborreliose, Karditiden) und später chronifizieren. In Abhängigkeit vom Stadium und der klinischen Ausprägung der Erkrankung wird eine 2- bis 3-wöchige Antibiotikatherapie mit Doxycyclin oder Ceftriaxon empfohlen. Unabhängig von einer durchgemachten Borreliose kann jeder neue Zeckenbiss jedoch wieder zu einer Neuinfektion führen.

Auch Infektionen nach Insektenstichen sind gerade im Kleinkindesalter häufig zu beobachten, so z. B. durch Hautflügler, wozu Bienen und Wespen gehören, aber auch Zwei- flügler, z. B. Stechmücken und Bremsen. Bei multiplem Auftreten dieser Stiche muss differenzialdiagnostisch an Varizellen gedacht werden. Im Rahmen so genannter Urlaubsdermatosen sind auch exotische Krankheiten in die Differenzialdiagnose unklarer Weichteilinfektionen einzubeziehen. Die Myiasis migrans (Hautmaulwurf) wird durch Eindringen von Larven bestimmter Stechmücken (Drasselfliege) in die Haut erzeugt. Die Larven (Larva migrans) wandern in gewundenen Gängen durch die Haut.

\subsection{Helminthen}

In die Differenzialdiagnose bestimmter Hauterkrankungen bzw. Weichteilinfektionen sind auch Helminthen, d. h. Würmer einzubeziehen.

\section{$3 \quad$ Entzündungsausbreitung}

Hinsichtlich der Entzündungsausbreitung von Weichteilinfektionen unterscheiden wir einmal die lokale Aussaat, wie den Abszess, das Empyem, die Phlegmone und die Lymphangitis sowie die systemische Ausbreitung. Letztere wird charakterisiert durch die Pyämie bzw. die Sepsis.

\subsection{Lokale Entzündung}

\section{Abszess}

Der Abszess grenzt sich gegen die Umgebung durch eine Granulationsschicht ab. Beim Abszess sollte nach der 
Inzision gerade bei Kindern nach Fremdkörpern gesucht und diese entfernt werden, was sich teilweise schwierig gestalten kann und zu mehrfachen Revisionen zwingt. Eine Drainage des Abszesses ist zu empfehlen. Eine Antibiotikagabe ist in der Regel nicht erforderlich. Der periproktische Abszess sollte radiär bis zum Darm inzidiert werden. Er entsteht häufig im Gefolge von inkompletten Analfisteln, die gleichzeitig mit exzidiert werden sollten. Differenzialdiagnostisch ist bei älteren Kindern auch an einen Morbus Crohn zu denken. Die abszedierende Lymphadenitis coli muss inzidiert und gegeninzidiert werden. Eine zusätzliche Drainage ist sinnvoll. Gegen Staphylokokken wirksame Antibiotika sollten je nach Ausdehnung des Befundes gegeben werden. Die Mastitis des Neugeborenen erfordert primär eine lokale antiseptische Behandlung, unter Umständen eine vorsichtige radiäre Inzision. Eine Antibiotikatherapie sollte durchgeführt werden.

\section{Phlegmone}

Die Phlegmone ist eine Infektion, bei der die Zwischenräume des Bindegewebes infiltriert werden und die auf diesem Weg auch fulminant fortschreiten kann. Sie grenzt sich im Allgemeinen nicht durch eine Membran ab. Häufigste Erreger sind im Gegensatz zum Abszess hier die Streptokokken. Letztere bilden Hyaluronidase und Streptokinase, wodurch die flächenhafte Ausdehnung der Entzündung gefördert wird. Die Phlegmone muss inzidiert und mit Drainagen versehen werden, wobei auch eine mögliche Abszedierung abgewartet werden kann. Eine Immobilisierung und eine Antibiotikatherapie sind erforderlich.

\section{Empyem}

Ein Empyem stellt eine Eiteransammlung in anatomisch präformierten Hohlräumen dar, wie z. B. der Pleura (s. unten), der Gallenblase oder auch einem Gelenk. Neben der gezielten antibiotischen Therapie steht die chirurgische Entlastung durch Punktion oder Drainage, ggf. auch durch eine Spülung im Vordergrund.

\section{Lymphangitis}

Bei der Lymphangitis breitet sich die Entzündung (,roter Streifen") entlang der Lymphbahnen infolge einer infizierten Verletzung der Füße oder Hände aus. Die Gefahr einer systemischen Ausbreitung mit Bakteriämie und Sepsis ist möglich. Deshalb ist hier primär eine antibiotische Behandlung indiziert, z. B. mit einem Breitband-Cephalosporin.

\subsection{Systemische Entzündung}

Eine Bakteriämie (Pyämie) ist die Folge einer passageren Einschwemmung von Bakterien in das Blut ohne allgemeines Krankheitsgefühl, kann aber von Schüttelfrost begleitet werden. Bei der Sepsis dagegen vermehren sich die Keime im strömenden Blut, v. a. im Kapillargebiet. Die Folgen der Sepsis werden im Rahmen der Peritonitis dargestellt (Abschn. 4.5).

\section{$4 \quad$ Infektionsarten}

\subsection{Weichteilinfektionen}

\section{Pyodermie der Haut}

Als bakterielle Pyodermien der Haut sind die kleinblasige Impetigo contagiosa, auftretend an asymmetrischen Körperstellen, sowie ihre bullöse Form zu nennen. Beide sind hoch infektiös. Eine lokale Behandlung genügt in der Regel. Antibiotika sind nur bei ausgedehntem Befall (Staphylokokken, selten Streptokokken) notwendig. Als Sonderformen gelten das staphylogene Pemphigoid bzw. das Lyell-Syndrom.

\section{Pyodermie der Haarfollikel}

Betreffen die Pyodermien die Haarfollikel, sprechen wir von einer Osteofollikulitis, die mit Pusteln einhergeht. Schreitet eine solche Osteofollikulitis fort, d. h. die Erreger dringen tief in den Haarfollikel ein und führen zur nachfolgenden Einschmelzung, sprechen wir von einem Furunkel. Eine Furunkulose liegt vor, wenn chronisch-rezidivierend über Monate und Jahre Furunkel in multiplen Lokalisationen auftreten. Hier muss an einen Diabetes mellitus, an eine chronische Nephritis, aber auch an eine primäre Immunschwäche gedacht werden. Treten Furunkel im Gesicht oberhalb der Oberlippe auf, befinden sie sich im Abfluss der V. angularis und V. ophthalmica mit Verbindung zum venösen Sinus des Gehirns. Prinzipiell müssen Furunkel inzidiert und der zentrale Nekrosepfropf entfernt werden. Der Gesichtsfurunkel sollte stationär behandelt werden. Hier sind lokale Manipulationen weitgehend zu vermeiden. Ein Kauverbot mit einer zusätzlichen Antibiotikagabe ist notwendig. Konfluieren mehrere benachbarte Furunkel, sprechen wir von einem Karbunkel. Hier muss neben der lokalen Behandlung dieser Karbunkel exzidiert werden.

\section{Erysipel}

Ein Erysipel (Wundrose) wird durch Streptokokken der Gruppe A, selten der Gruppe G verursacht. Die Erreger breiten sich in den Lymphspalten der Dermis aus. Die Krankheit verläuft mit Ausbildung von asymmetrischen, gleichförmigen Erythemen, die eine bogige Begrenzung mit Überwärmung und ödematöser Schwellung aufweisen. Das Erysipel soll mit Bettruhe, lokal antiseptischen Verbänden und einer systemischen Antibiotikatherapie (Penicillin) behandelt werden.

\section{Panaritium}

Panaritien sind pyogene Infektionen an der Palmarseite der Finger. Je nach Tiefenausdehnung unterscheiden wir verschiedene Formen des Panaritiums: 
- Panaritium cutaneum,

- Panaritium subcutaneum,

- Panaritium tendinosum, wobei bei Befall des I. und des V. Fingers infolge der besonderen Anatomie eine V-Phlegmone entstehen kann,

- Panaritium articulare,

- Panaritium ossale.

Panaritien mit Einschmelzung benötigen ein chirurgisches Vorgehen mit Inzision und ggf. einer Lascheneinlage.

\section{Paronychie}

An der Dorsalseite treten häufig Paronychien auf. Es besteht eine Eiterstraße entlang des Hautrandes der Fingernägel (Umlauf). Je nach Ausdehnung und v. a. bei Einschmelzung sollte die Paronychie inzidiert und der Nagel entfernt werden. Gelegentlich kann eine Antibiotikabehandlung erforderlich sein.

\subsection{Nosokomiale Infektionen}

Unter einer nosokomialen Infektion wird eine Infektion verstanden, die zum Zeitpunkt der stationären Aufnahme in ein Krankenhaus nicht manifest oder inkubiert war und die frühestens $48 \mathrm{~h}$ nach Aufnahme erste Symptome zeigt.

Sowohl bei Erwachsenen als auch bei Kindern stellt sie eine schwere Komplikation während einer stationären Behandlung dar. Sie erhöht substanziell die Morbidität der Patienten, verlängert den stationären Aufenthalt, erhöht die Behandlungskosten und stellt letztlich einen nicht unwesentlichen Faktor für die Mortalitätsrate in einem Krankenhaus dar.

\section{Epidemiologie}

In Europa und den USA sind zwischen 5 und $10 \%$ aller stationär behandelten, erwachsenen Patienten von einer nosokomialen Infektion betroffen. Allgemein wird davon ausgegangen, dass die Frequenz nosokomialer Infektionen im Kindesalter niedriger ist. In einer aktuellen europäischen Multicenterstudie liegt diese bei 2,5\% und ist damit mit denen in Kanada und den USA vergleichbar. Bezogen auf das Alter der Kinder stellt sich jedoch keine homogene Verteilung dar, sondern es besteht eine reziproke Korrelation zwischen Infektionshäufigkeit und Alter. Jenseits des 10. Lebensjahrs liegt die Frequenz zwischen 1,5 und $4 \%$, im Säuglingsalter dagegen zwischen 7 und $9 \%$. Das größte Risiko für das Auftreten einer nosokomialen Infektion (bis zu $30 \%$ ) haben Frühgeborene mit einem Geburtsgewicht $<800$ g, aber auch reife Neugeborene mit einem zentralen Venenkatheter (Raymond et al. 2000).
Im Wesentlichen werden im Kindesalter fünf verschiedene Typen von nosokomialen Infektionen beobachtet:

- Bakteriämie, meist im Zusammenhang mit einem zentralen Venenkatheter (35\%),

- Infektionen der unteren Atemwege (33\%),

- Harnwegsinfekte (11\%),

- postoperative Wundinfekte (7\%),

- gastrointestinale Infekte (14\%).

Dabei ist jedoch $\mathrm{zu}$ beobachten, dass die Frequenzen der jeweiligen Infektionen sowohl zwischen verschiedenen Krankenhäusern als auch zwischen den jeweiligen pädiatrischen Abteilungen eine große Variabilität zeigen (Raymond et al. 2000).

In einer retrospektiven Analyse einer kinderchirurgischen Abteilung, in der an 537 Kindern 575 Operationen durchgeführt wurden, lag die Rate der postoperativen Wundinfektionen bei $6,7 \%$. Aseptische Operationen hatten dabei eine Infektionsrate von $2,7 \%$, während diese bei den unsauberen Operationen auf 14,6 \% anstieg. Interessanterweise war die Infektionsrate beispielsweise unabhängig von einer perioperativen Antibiotikaprophylaxe. Es bestand lediglich ein signifikanter Zusammenhang mit der Länge der Operation, dem Kontaminationsgrad und dem Ausbildungsstand des Operateurs (Duque-Estrada et al. 2003).

\section{Erregerspektrum}

Als Erreger nosokomialer Infektionen werden in der überwiegenden Mehrzahl Bakterien nachgewiesen. Aber auch Viren (Rotavirus, respiratorisches Synzytial-Virus, Zytomegalie-Virus, Adenovirus, Norovirus) sind insbesondere bei pulmonalen und gastrointestinalen Infektionen zu finden. Außerdem gewinnt Candida albicans als Ursache für Mykosen eine zunehmende Bedeutung. Unter den bakteriell bedingten nosokomialen Infektionen werden bei den postoperativen Wundinfektionen in der überwiegenden Zahl Staphylococcus aureus nachgewiesen (12-37\%), aber auch koagulasenegative Staphylokokken, Enterobacter cloacae sowie Pseudomonas aeruginosa. Dagegen sind für die katheterassoziierten Bakteriämien in der Hälfte aller Fälle koagulasenegative Staphylokokken und für die Infektion der tieferen Atemwege in $35 \%$ Pseudomonas aeruginosa verantwortlich.

\section{Antibiotikaassoziierte Diarrhoe}

Eine in den letzten Jahren zunehmende Bedeutung haben gastrointestinale Infektionen durch Clostridium difficile, das in bis $\mathrm{zu} 20 \%$ aller antibiotikaassoziierten Diarrhoen als ursächlich angesehen werden muss. Die Infektionsrate korreliert mit der Dauer der Antibiotikatherapie und prinzipiell stellt jedes Antibiotikum ein potenzielles Risiko für eine Clostridium-difficile-Infektion dar. Daher muss die Indikation 
zu einer Antibiotikatherapie streng gestellt und das optimale Antibiotikum ausgewählt werden. Dazu sollte nicht nur die aktuelle Resistenzlage der häufigsten Keime Berücksichtigung finden, sondern auch das jeweilige Keimspektrum einschließlich Resistenzlage, beispielsweise auf Intensivtherapiestationen, bekannt sein.

\section{Methicillinresistente Staphylococcus aureus}

Eine Sonderform nimmt die Infektion mit dem methicillinresistenten Staphylococcus aureus (MRSA) ein. Hier sind spezielle therapeutische und prophylaktische Maßnahmen vorzunehmen. Es muss eine räumliche Isolierung des Patienten erfolgen. Die Untersuchung bzw. die Pflege des Patienten sind mit Kitteln und Handschuhen durchzuführen, die im Zimmer des Patienten verbleiben. Unter bestimmten Umständen (nasopharyngeale Kolonisation der MRSA) muss Mundund Nasenschutz getragen werden. Es ist eine tägliche Wischdesinfektion der Kontaktgegenstände mit den vorgeschriebenen Flächendesinfektionslösungen durchzuführen und die Wäsche- und Müllentsorgung erfolgt in separaten Sammelbehältern im Patientenzimmer. Eine Antibiotikabehandlung sollte nur bei klinischen Anzeichen einer Infektion durchgeführt werden. Unabhängig davon muss der Patient einmal täglich mit antiseptischer Seife einschließlich der Kopfhaare gewaschen werden. Sobald es der Zustand des Patienten erlaubt, sollte dieser aus dem Krankenhaus entlassen werden. Das Auftreten der MRSA muss deutlich sichtbar in der Patientenakte vermerkt und ein mögliches Zielkrankenhaus bei einer Verlegung im Vorfeld informiert werden. Abschließend sollte eine Schlussdesinfektion des Patientenzimmers erfolgen.

Infektionen mit MRSA-Stämmen gewinnen rapide an Bedeutung. In den USA sind aktuell bereits $20 \%$ aller nosokomialen Bakteriämien durch MRSA hervorgerufen. Dies erfordert neue Strategien insbesondere bei der adjuvanten Antibiotikatherapie, wobei die Frage nach dem optimalen Antibiotikum diskutiert werden muss. Aber auch die Einführung Antibiotika, Antiseptika oder Metallionen beschichteter Kathetersysteme wird diskutiert, wodurch beispielsweise katheterassoziierte Bakteriämien bis zu 90 \% reduziert werden können.

Analog zum MRSA existieren zunehmend multiresistente gramnegative Bakterienstämme (MRGN), die u. a. durch verstärkte Bildung von $\beta$-Laktamasen charakterisiert sind und daher früher als ESBL-Keime (Extended Spectrum Beta Laktamase) bezeichnet wurden. Aktuell werden sie entsprechend ihrer Resistenz auf die vier existierenden Antibiotikaklassen charakterisiert. Klinisch relevant sind dabei die 3MRGN und 4MRGN-Bakterienstämme, wobei letztere gegenüber allen Antibiotikaklassen resistent sind. Sie stellen für die Krankenhaushygiene ein zunehmendes Problem dar und sind häufig die Ursache für infektiöse Komplikationen insbesondere auf neonatologischen Intensivtherapiestationen (Laux et al. 2013).

Es gelten vergleichbare Hygienevorschriften wie für die MRSA-Infektionen, jedoch ist eine Isolation der Patienten nur auf Intensivtherapiestationen mit Nachweis der Erreger im Respirationstrakt oder in der Umgebung von immunsupprimierten Patienten notwendig.

Letztlich sind elementare hygienische Maßnahmen die wesentlichste Grundlage zur Vermeidung von nosokomialen Infektionen.

So sollten im Säuglingsalter nur hitzesterilisierte Sauger und Glasflaschen Verwendung finden. Zubereitete Flaschennahrung wird unverzüglich gefüttert. Reste werden verworfen, um einer Keimvermehrung und somit dem Infektionsrisiko vorzubeugen. Und schließlich konnte gezeigt werden, dass eine optimale Händedesinfektion auf Intensivstationen zur Reduzierung von katheterassoziierten Bakteriämien um $25 \%$ geführt hat (Wenzel 2007).

\subsection{Katheterinfektionen}

\section{Definition}

Im Kindesalter spielen Infektionen zentralvenöser Katheter, aber auch von Shunts bei ventrikulo-peritonealen bzw. ventrikulo-atrialen Drainagen sowie externer Ventrikeldrainagen eine bedeutende Rolle. Im Rahmen von Qualitätsmanagementmaßnahmen ist die Erfassung von katheterassoziierten Infektionen ein etabliertes Instrument. Bei Verdacht auf eine ZVK-assoziierte Infektion sollten mindestens 2 Blutkulturpärchen - die erste über den Katheter und die zweite über eine periphere Vene - entnommen werden. Eine Katheterinfektion kann als gesichert gelten, wenn eine positive Blutkultur aus dem zentralen Katheter bei gleichzeitig negativer, zeitlich verzögerter positiver oder mit geringer Keimzahl positiver Blutkultur aus einer peripheren Vene oder mindestens zwei peripheren Blutkulturen nachgewiesen werden kann.

\section{Diagnostik}

Als zuverlässigste Methode zur Diagnostik einer katheterassoziierten Blutstrominfektion gilt die quantitative Blutkulturdiagnostik. Sie ist aber aufwendig und relativ teuer und leider in den meisten Laboratorien nicht etabliert. Als relativ neue Methode zur In-situ-Diagnostik wird daher die „differential time to positivity“ (DTP-Methode) favorisiert, wobei nach oben beschriebener Entnahme von Blutkulturen die Zeit bis zum positiven Wachstumssignal in Blutkulturautomaten ermittelt wird (Seifert et al. 2007).

Bei Entfernung bzw. Wechsel des zentralvenösen Katheters sollte die Katheterspitze nur bei Verdacht auf eine Katheterinfektion zur mikrobiologischen Untersuchung eingesandt werden, da sehr häufig beim Ziehen die Spitze des Katheters kontaminiert wird. Folglich korreliert ein Keimnachweis keinesfalls mit einer vorliegenden Infektion. 


\section{Katheterwechsel}

Zur Vorgehensweise gibt es eindeutige Empfehlungen der Deutschen Gesellschaft für Krankenhaushygiene und Infektiologie (Scholz et al. 2003). Ein routinemäßiger Wechsel von zentralen Venenkathetern senkt nicht das Risiko einer katheterinduzierten Sepsis. Deswegen ist bei jeglicher Versorgung mit solchen Kathetern auf Asepsis und Sterilität zu achten. Infusionssysteme werden nach den aktuellen HCPACRichtlinien im Intervall von etwa $72 \mathrm{~h}$ gewechselt, außer bei anderer klinischer Indikation. Dagegen sollten Infusionsschläuche, über die Blutprodukte oder Fettlösungen verabreicht werden, innerhalb von $24 \mathrm{~h}$ gewechselt werden. Antibiotika zur Prophylaxe einer Kathetersepsis vor dem Legen oder während des Gebrauchs werden in der Regel nicht verabreicht - außer bei immunsupprimierten Patienten (Abschn. 4.4).

\section{Therapie}

In der Therapie katheterassoziierter Infektionen werden die lokalen Infektionen mit Rötung, Induration und Exsudation an den Katheteraustrittsstellen von den sog. „Tunnelinfektionen" mit der Rötung entlang des subkutanen Kanals unterschieden. Bei ersteren reicht in der Regel eine lokale Wundbehandlung aus. Im Einzelfall muss über eine systemische Antibiotikagabe entschieden werden. Bei einer Infektion entlang des subkutan liegenden Katheters sollten generell systemische Antibiotika unter klinischer und laborchemischer Kontrolle, später entsprechend des Keimspektrums, verabreicht werden. Sind die o. g. Maßnahmen nicht erfolgreich, so muss der Katheter entfernt werden (Fätkenheuer et al. 2002; Mermel et al. 2001). Mit der Implantation neu entwickelter, mit Silber oder Antibiotika imprägnierter Katheter, die die Adhärenz von Mikroorganismen und die Proliferation an der Katheterwand verringern sollen, könnte dagegen nach neueren Studien die Infektionsrate gesenkt werden. Außerdem hat eine aktuelle Metaanalyse gezeigt, dass ebenfalls die Blockung zentraler Katheter mittels Ethanol- oder Vancomycinlösung sowie die Verwendung von mit Chlorhexidin imprägnierten Wundverbänden als Infektionsprohylaxe effektiv sind (Carbon et al. 1999; Huang et al. 2011).

\section{Liquor-Shunt-Operation}

Bei Liquor-Shunt-Operationen existieren derzeit keine gesicherten Studien, die zur perioperativen Antibiotikaprophylaxe eindeutig Stellung beziehen. Eine englische Studie aus dem Jahr 1994 (Brown et al. 1994) empfiehlt die antimikrobielle Prophylaxe, andere Studien dagegen zeigten, dass mit einer solchen Prophylaxe - sei sie systemisch oder lokal subkutan verabreicht - eine Ventrikulitis und damit eine Katheterinfektion nicht signifikant verhindert werden konnte. Dagegen wird bei Anlage einer externen Ventrikeldrainage entweder primär bei hohen Liquoreiweißwerten oder nach Shunt-Infektionen eine Antibiotikagabe empfohlen - auch wenn damit eine spätere Ventrikelinfektion nicht immer verhindert, wohl aber die Wundinfektionsrate gesenkt werden kann. Analog dazu konnte tierexperimentell gezeigt werden, dass durch die Verwendung silberbeschichteter Kathetersysteme sowohl die katheterassoziierte Infektionsrate als auch die entzündliche Reaktion des periventrikulären Hirnparenchyms gesenkt werden kann (Hazer et al. 2012).

\subsection{Infektionen bei immunsupprimierten Patienten}

\section{Neutropenie}

Die Neutropenie (drohender Abfall der Neutrophilen $<500$ Granulozyten $/ \mathrm{mm}^{3}$ ) ist der wichtigste Risikofaktor für das Auftreten einer Infektion bei immunsupprimierten Patienten, wobei die Gefahr mit der Dauer der Neutropenie ( $>10$ Tage beginnt ein hohes Risiko) steigt. Gleichzeitig auftretendes Fieber spricht in der Regel für eine solche Infektion, wobei die Zeichen einer Inflammation nur selten ausgeprägt sind, unter Umständen sogar völlig fehlen können (z. B. Fehlen ausgeprägter Infiltrationen im Röntgenbild des Thorax, Fehlen des Erythems oder Induration der Haut). Weitere Risikofaktoren für das Auftreten einer Infektion sind eine veränderte Granulozytenfunktion, besonders der Phagozytose, Defekte im Bereich der normalen physikalischen Abwehrbarrieren wie Haut und Schleimhäute, pathologische Veränderungen der endogenen Mikroflora, notwendige zentrale Venenkatheter (Abschn. 4.3) und eine ausgebliebene Remission der Grundkrankheit. Primäre Infektionen werden überwiegend durch grampositive und gramnegative Bakterien, selten durch Anaerobier oder Viren verursacht. In der Phase länger dauernder Neutropenien im Verlauf der Therapie einer Grundkrankheit spielen v. a. Pilzinfektionen (Aspergillose, Kandidose) eine bedeutende Rolle (Abb. 2).

\section{Therapie}

Die Standardtherapie bei fiebernden immunsupprimierten Patienten ist die unverzügliche intravenöse Antibiotikagabe. Das gilt auch für fieberfreie Patienten, die andere Zeichen einer Infektion aufweisen. Bei Hochrisikopatienten sind im Gegensatz zu Niedrigrisikopatienten schwerere Verläufe auch unter entsprechender Therapie zu erwarten. Deswegen sind strenge Richtlinien zur Infektionsprophylaxe bei immunsupprimierten und somit auch bei pädiatrischonkologischen Patienten unverzichtbar. Diese betreffen Empfehlungen zur nicht medikamentösen, antibakteriellen, antimykotischen und antiviralen Prophylaxe sowie zur Infektionsprävention durch Einsatz hämatopoetischer Wachstumsfaktoren und schließt die Impfempfehlungen für die genannte Patientengruppe ein (Graubner et al. 2001). Operative Eingriffe im Rahmen der Grundkrankheit sollten bei einer Neutropenie $<1000 / \mathrm{mm}^{3}$ nicht vorgenommen werden. 


\subsection{Peritonitis}

\section{Definition}

Im Vordergrund der septischen Bauchchirurgie steht das Krankheitsbild der Peritonitis. Sie stellt per definitionem eine diffuse oder lokalisiert auftretende, in der Regel akut verlaufende Entzündung des Peritoneums dar, die meist bakteriell, aber auch chemisch-toxisch bedingt sein kann. Demnach gehört die Peritonitis zum klinischen Bild des akuten Abdomens. Im eigentlichen Sinn handelt es sich bei der Peritonitis nur um einen Sammelbegriff für ätiologisch, pathophysiologisch und morphologisch unterschiedliche Erkrankungen.

\section{Einteilung}

Nach der Ätiologie unterscheiden wir im Kindesalter eine primäre, eine sekundäre, eine chemische und eine physikalische Form, nach der Ausbreitung eine lokale, die zur Abszedierung führen kann, eine auf den Unterbauch begrenzte oder im schwersten Fall eine diffuse im ganzen Bauchraum ausgebreitete Peritonitis. Das dabei vorhandene Exsudat kann sowohl serös, fibrinös, purulent, hämorrhagisch, putrid, gallig, mekoniumhaltig bzw. kotig je nach Ursache bzw. Alter des Kindes sein.

\section{Pathophysiologie}

Über die enorme Resorptions- und Transsudationsfähigkeit sowie die biologische Barriere des Peritoneums wurde schon Ende des 19. Jahrhunderts von Wegener berichtet. Damit wurden auch schon Therapieschemata festgelegt, die im Grundsatz heute noch Gültigkeit haben. Infolge der Infektion reagiert der Organismus mit einer systemischen Entzündungsreaktion (systemic inflammatory response syndrome - SIRS), die bei Ausbreitung der Infektion zu einer Sepsis führt. Bakterien und bakterielle Stoffwechselprodukte (Endotoxine) bewirken nach Kontakt mit Leukozyten oder Endothelzellen eine Aktivierung derselben, die dann Mediatoren wie Prostaglandine, Elastase, Sauerstoffradikale, Histamine, Kinine und andere Substanzen freisetzen. Diese führen zu systemischen Effekten wie Fieber sowie vermehrtem Volumenbedarf bei weit gestellter Gefäßperipherie. Wir sprechen dabei von einem distributiven Schock, der über die inflammatorische Aktivierung der Gerinnungskaskade zur disseminierten intravasalen Koagulation (DIC), zum akuten Atemnotsyndrom (ARDS) und letztendlich zum septischen Schock mit einem Multiorganversagen führen kann. Vieles ist noch unklar im Zusammenwirken der Mediatoren, v. a. auch, welche Faktoren zum Nachweis einer Sepsis geeignet sind. Sensibler als die Thrombozyten reagieren die Gerinnungsfaktoren wie Fibrinogen und besonders Antithrombin III, die frühzeitig eine anlaufende Verbrauchskoagulation anzeigen.

Als primäre Peritonitis wird die Infektion der Abdominalhöhle durch eine hämatogene Aussaat der Erreger in das
Abdomen verstanden. Wichtigste Formen sind die Pneumound Streptokokkenperitonitis.

Die Hauptursachen der sekundären Peritonitis im Kindesalter sind:

- Durchwanderungsperitonitis,

- Perforationsperitonitis,

- aszendierende Entzündung bei großen Mädchen.

\section{Verlauf}

Der Verlauf einer Peritonitis im Kindesalter ist durch eine frühe proinflammatorische Allgemeinreaktion und eine $a b$ dem zweiten Erkrankungstag gezielt einsetzende antiinflammatorische Reaktion gekennzeichnet. Aus diesem Grund ist im Kindesalter der septische Schock mit einem folgenden tödlichen Ausgang eher eine Rarität.

Die häufigste Ursache einer sekundären Peritonitis im Kindesalter stellt die Appendicitis perforata dar.

\section{Therapie}

Wichtig sind eine frühzeitige Diagnostik einer jeden Form der Peritonitis sowie die schnelle Beseitigung ihrer Ursache, um ein Fortschreiten der Peritonitis zu verhindern. Ist die Diagnose einer Peritonitis gestellt, sind präoperativ Maßnahmen einzuleiten, die den Zustand des Kindes stabilisieren. Bestehende Elektrolytveränderungen, Störung des SäureBasen-Haushalts und ein Volumenmangel sind zu beseitigen. Im Vordergrund der Behandlung der Peritonitis steht die operative Therapie begleitet von konservativen Maßnahmen. Die Grundsätze der septischen Bauchchirurgie liegen in der Beseitigung der Infektionsursache und der Entfernung des entzündlichen Exsudats. Erfolgt die Abklärung der Infektionsursache nicht laparoskopisch, sollte die Schnittführung für eine gute Übersicht im Abdomen sorgen. Nur bei eindeutigem Nachweis einer perforierten Appendizitis ist dann ein Schnitt im rechten Unterbauch erlaubt. Wenn im Ausnahmefall Drainagen gelegt werden (z. B. bei der diffusen Peritonitis), dürfen diese nicht aus der Wunde herausgeleitet werden.

Cave: Bei einer nachgewiesenen Nahtinsuffizienz oder Perforation eines Darmabschnitts, außer bei der Appendix oder eines Meckel-Divertikels, sollten primäre Anastomosen vermieden werden. Besser ist eine direkte Vorlagerung des betroffenen Darmabschnitts oder im Einzelfall auch eine vorgeschaltete Darmfistel. Eine innere Schienung zwecks lleusprophylaxe ist wegen der Vulnerabilität der entzündeten Darmwand abzulehnen.

Die zusätzliche konservative Therapie im Rahmen der postoperativen Phase beinhaltet eine intensivmedizinische Behandlung mit einer zunächst unspezifischen kombinierten Antibiotikagabe der in der Regel vorliegenden Mischinfektion 
von aeroben und anaeroben Keimen. Besonderes Augenmerk sollte auf die Antithrombin-III-Substitution gelegt werden.

Nach Kenntnis des Erregerspektrums und des Resistogramms muss dann unverzüglich in eine gezielte Antibiotikagabe gewechselt werden.

\section{Komplikationen}

Als Komplikationen der Peritonitis sind zu nennen:

- Abszedierungen der Bauchdecke und des Douglas-Raum,

- Interenterische Abszesse (sog. Schlingenabszess), subphrenische und subhepatische Abszesse,

- Nahtinsuffizienzen mit entsprechenden Stuhlfisteln.

Als schwerste systemische Komplikation gilt die Sepsis, die wie bereits dargestellt, zum septischen Schock und Multiorganversagen führen kann. Im Gegensatz zum Erwachsenen ist Letzteres im Kindesalter äußerst selten.

Infolge erheblicher Adhäsionen, aber auch Bridenbildungen, können Subileuserscheinungen bis hin zum Vollbild des Ileus Monate bis Jahre später auftreten. Bei weiblichen Patienten sind Verklebungen im Bereich der Fimbrien der Tube möglich, die sehr oft $\mathrm{zu}$ einer späteren Sterilität führen können.

\subsection{Pleuraempyem}

\section{Definition}

Das Pleuraempyem stellt eine Ansammlung von Eiter oder putrider Flüssigkeit in der anatomisch präformierten Pleurahöhle bei fehlender primärer Gewebenekrose dar. Der $\mathrm{pH}-$ Wert des Empyems ist obligat $<7$.

\section{Ätiologie \\ Als Ursache für ein Pleuraempyem wird im Kindesalter meist die para- oder metapneumonische Ruptur eines Lungenabs- zesses beobachtet. Aber auch postoperativ bei Stumpfinsuf- fizienz einer Lungenresektion oder Anastomoseninsuffizi- enz einer Ösophagusanastomose, bei Superinfektion eines Hämatothorax oder bei Ausbreitung eines entzündlichen Oberbauchprozesses (beispielsweise ein subphrenischen Abszesses) kann ein Pleuraempyem auftreten.}

\section{Klinik}

Die Patienten zeigen die typischen Symptome einer schweren systemischen Infektion verbunden mit einer ausgeprägten Dyspnoe, Klopfschalldämpfung und Abschwächung des Atemgeräusches auf der Seite der erkrankten Lunge. Im Verlauf bildet sich häufig eine Pleuraschwarte aus, die zu einer restriktiven Atemfunktionsstörung führt. Das Pleuraempyem kann per continuitatem die Thoraxwand oder das Diaphragma infiltrieren und durchbrechen.

\section{Therapie}

Präoperativ ist die Darstellung in einem Schnittbildverfahren zu empfehlen (CT, MRT). Ziel der chirurgischen Intervention ist die komplette Entfernung des Eiters. Dies muss stadiengerecht erfolgen, wobei beim rein exsudativen Empyem allein das Legen einer Pleuradrainage möglich ist. Dagegen werden beim fibrinös-purulenten Empyem (septiertes Empyem) eine thorakoskopisch assistierte Beseitigung der Septen und die gezielte Einlage von Pleuradrainagen notwendig. Beim im Kindesalter selten vorkommenden organisierten Empyem (Plueraschwarte) ist eine Dekortikation meist über eine kleine Thorakotomie notwendig, um so die volle Wiederentfaltung der Lunge zu ermöglichen.

Begleitet wird dieses Vorgehen durch eine hoch dosierte Antibiotikatherapie nach Resistogramm und durch physiotherapeutische Maßnahmen, die die Belüftung der Lunge verbessern.

\section{$5 \quad$ Grundsätze der chirurgischen Infektionsbehandlung \\ Der schon seit Jahrhunderten bekannte Leitsatz „ubi pus ibi evacua" stellt unverändert das therapeutische Grund- konzept bei jeder Infektion dar. Das heißt, jede eitrige Infektion sollte chirurgisch behandelt werden, notfalls mit einer Inzision bzw. Exzision mit Beseitigung der Infek- tionsursachen. Primäre Wundverschlüsse sind bei allen Infektionen zu vermeiden.}

Wenn der Verdacht besteht, dass sich in der Wunde ein Fremdkörper befindet, muss dieser gesucht und entfernt werden. Ein Wundabstrich für den mikrobiologischen Nachweis von Erregern ist unbedingt erforderlich. Ein primärer kompletter Wundverschluss sollte nicht vorgenommen werden, um die Bildung von subkutanen Eiteransammlungen zu verhindern. Es ist durchaus auch möglich, eine Wunde mit dem Einlegen einer Drainage offen zu halten. Eine Ruhigstellung der betroffenen Extremität in einem Schienenverband ist erforderlich. Sind Teile des Gesichts bzw. der Stirn betroffen, ist Bettruhe einzuhalten. Analoge Prinzipien gelten für die Behandlung einer Lymphangitis.

Die lokale Wundbehandlung ist durch regelmäßige Verbandswechsel fortzusetzen, wobei die Säuberung der Wunde Grundlage einer sekundären Wundheilung darstellt. Gegebenenfalls besteht die Indikation zur Durchführung einer sog. Vac-Therapie. Dabei wird durch eine luftdichte Versiegelung eines Wundgebiets mit gleichzeitig pulsativ angelegtem Unterdruck kontinuierlich über dem gesamten Wundgebiet Wundsekret abgesaugt. Dadurch wird die Wunde gereinigt, aber gleichzeitig die Durchblutung verbessert und somit die körpereigene lokale Infektabwehr stimuliert sowie die Wundheilung gefördert. Eine systemische Antibiotikagabe ist bei 
jeder ausgedehnten lokalen Infektion zu empfehlen. Diese erfolgt zunächst adjuvant und muss ggf. später entsprechend des Resistenzspektrums der Erreger angepasst werden. Bei Verdacht auf Virus- bzw. Pilzinfektionen ist eine entsprechende antivirale bzw. antimykotische Therapie lokal ggf. auch als systemische Behandlung notwendig. Auf die Vollständigkeit der Impfung, insbesondere der Tetanus-Schutzimpfung ist zu achten. Notfalls muss eine passive Immunisierung erfolgen. Spezielle Maßnahmen sind bei MRSA- oder HIV-infizierten Patienten entsprechend der Hygieneordnung einzuhalten.

\section{Prävention von Infektionen}

\subsection{Chirurgische Wundversorgung}

Neben der Beachtung von Asepsis und Antisepsis, wozu auch die hygienischen Maßnahmen, die Desinfektion und die Sterilisation gehören, ist die Einhaltung etablierter chirurgischer Techniken bei der Prävention von Infektionen von großer Bedeutung, so z. B. die typische Friedrich-Wundversorgung bei der Notfallversorgung. Weiterhin ist auf einen richtigen adaptierenden Wundverschluss zu achten. Um eine ungestörte Wundheilung zu ermöglichen, sollten tägliche Verbandswechsel bei sog. aseptischen Wunden nicht vorgenommen werden. Allgemeine hygienische Maßnahmen, die in einer jeweils gültigen Hygieneordnung der Einrichtung fixiert sind, müssen in der Klinik bzw. auch in der Praxis eingehalten werden.

\subsection{Antibiotikaprophylaxe}

Ein wesentlicher Anteil der Antibiotikagabe in der Kinderchirurgie dient der Prophylaxe postoperativer Infektionen. Dazu gehören die präoperative, die perioperative sowie die postoperative Medikation, die in Abhängigkeit des vorgesehenen Eingriffs, der Lokalisation und des Alters der Patienten verabreicht werden muss. Im Vordergrund steht hierbei die perioperative Antibiotikagabe. Da im Kindesalter keine prospektiven Studien existieren, die eine Reduktion postoperativer Wundinfektionen zeigen könnten, werden wegen der ähnlichen Pathomechanismen des Auftretens von Wundinfektionen die bekannten Studien für Erwachsene berücksichtigt. Das Ziel einer solchen Antibiotikagabe soll die Reduktion bzw. Elimination von Keimen sein, die bei der Operation die Wunde kontaminieren und zu nachfolgenden Infektionen führen können.

Nosokomiale Infektionen, die die Lunge bzw. die Harnwege betreffen, werden dagegen nicht beeinflusst und sollten sowohl durch präoperative als auch postoperative Antibiotikagaben vermieden werden.
Das Risiko einer Infektionsentstehung und die Ausbreitung einer solchen ist besonders erhöht bei Kindern, bei denen Implantate aller Art eingebracht werden, bei immunsupprimierten Kindern und bei Kindern, bei denen kontaminierte Organe und Regionen bei der Operation eröffnet werden müssen. Zu letzteren gehören insbesondere Eingriffe am Dickdarm bzw. am Urogenitalsystem.

Primär aseptische Eingriffe, bei denen der bakterielle Kontaminationsgrad durch Einhaltung aller Hygienestandards nicht negativ beeinflusst wird, erfordern im Kindesalter keine Antibiotikaprophylaxe.

Der Erfolg einer perioperativen Antibiotikaprophylaxe wird einerseits durch den Zeitpunkt und die Zeitdauer der Applikation und andererseits vom gewählten Antibiotikum und dessen Dosis beeinflusst. Das benutzte Antibiotikum sollte dabei die häufigsten Infektionserreger in Abhängigkeit des vorgesehenen Eingriffs erfassen, aber auch gut verträglich für das Kind und kostengünstig sein. In der Regel erfolgt die Gabe über einen intravenösen Zugang. Um eine effiziente Konzentration des Antibiotikums sowohl im Blut als auch im Gewebe zum Zeitpunkt des operativen Eingriffs zu erreichen, sollte dieses etwa $30 \mathrm{~min}$ vor dem Hautschnitt appliziert werden. Bei längerer Operationsdauer sollte alle $4 \mathrm{~h}$ eine weitere Gabe vorgenommen werden, in der Regel aber nicht länger als $24 \mathrm{~h}$ (Mangram et al. 1999). Unter Umständen kann es aber durchaus sinnvoll sein, die Antibiotikatherapie in Abhängigkeit des klinischen Verlaufs und der Entzündungsparameter weiterzuführen.

\subsection{Endokarditisprophylaxe}

Es besteht die Hypothese, dass Bakteriämien, die im Rahmen medizinischer Eingriffe vorkommen, bei Patienten mit entsprechenden Risikofaktoren zu infektiösen Endokarditiden führen können. Dabei wird angenommen, dass prophylaktische Gaben von Antibiotika diese Erkrankungen effizient verhindern können. Die bisherigen Empfehlungen beruhen auf diesen theoretischen Überlegungen, auf Tierversuchen und Kasuistiken, auch wenn bekannt ist, dass mit einer solchen Prophylaxe nicht in jedem Fall eine Endokarditis wirkungsvoll verhindert werden kann. Das Ziel der bisherigen Leitlinien zur Prophylaxe der infektiösen Endokarditis war es, möglichst bei allen Patienten mit einem erhöhten Risiko die Entstehung einer infektiösen Endokarditis durch Bakteriämien im Zusammenhang mit medizinischen Eingriffen zu verhindern. Für die Effektivität und Effizienz dieses Vorgehens liegt nur eine unzureichende Evidenz vor.

In Anlehnung an die Empfehlungen der American Heart Association (AHA) orientieren sich die aktuellen Empfehlungen zur Endokarditisprophylaxe der Deutschen Gesell- 
schaft für Kardiologie und der Paul-Ehrlich-Gesellschaft für Chemotherapie allerdings mehr an der Frage, welche Patienten mit hoher Wahrscheinlichkeit von einer Antibiotikaprophylaxe profitieren werden. Unter diesem Aspekt ließe sich der bisherige Einsatz der Prophylaxe sinnvoll eingrenzen (Naber et al. 2007). Diesen aktuellen Empfehlungen kann jedoch zum jetzigen Zeitpunkt die Deutsche Gesellschaft für Pädiatrische Kardiologie nicht einheitlich folgen und so gelten momentan noch die bisherigen Standards. Danach werden Patienten mit einem erhöhten Risiko von Patienten mit einem hohen Risiko hinsichtlich einer bakteriellen Endokarditis unterschieden (s. Übersicht).

\section{Endokarditisrisiko}

- Patienten mit einem erhöhten Endokarditisrisiko:

- Die meisten angeborenen Herzfehler (außer Vorhofseptumdefekt vom Sekundum-Typ)

- Operierte Herzfehler mit einem Restbefund (ohne Restbefund $1 \mathrm{Jahr}$ )

- Hypertrophe obstruktive Kardiomyopathie

- Erworbene Herzklappenfehler

- Patienten mit einem hohen Endokarditisrisiko:

- Zustand nach mikrobieller Endokarditis

- Zyanotische Herzfehler

- Zustand nach Implantation einer Herzklappe (aus Kunststoff oder Biomaterial)

- Chirurgisch etablierte Shunts oder implantierte Konduits

Für diese Patienten sollte bei allen chirurgischen Interventionen einschließlich Endoskopien am Oropharynx, des Respirations- und Gastrointestinaltrakts, des Urogenitaltrakts sowie bei Eingriffen an Haut und Weichteilgeweben eine antibiotische Endokarditisprophylaxe erfolgen. Bei Patienten mit einem besonders hohen Endokarditisrisiko wird die Prophylaxe bereits bei einer nasotrachealen Intubation sowie bei Herzkatheteruntersuchungen empfohlen.

Eine Antibiotikaprophylaxe sollte generell $60 \mathrm{~min}$ vor einem Eingriff verabreicht werden (Tab. 1). Für Patienten mit einem erhöhten Endokarditisrisiko wird die einmalige präoperative Antibiotikaapplikation als hinreichend angesehen. Patienten mit einem hohen Endokarditisrisiko sollen bei

Tab. 1 Dosierung einiger empfohlener Antibiotika

\begin{tabular}{|c|c|c|}
\hline & Antibiotikum & Dosierung \\
\hline Orale Einnahme & Amoxicillin & $50 \mathrm{mg} / \mathrm{kg} \mathrm{KG}$ \\
\hline $\begin{array}{l}\text { Orale Einnahme nicht } \\
\text { möglich }\end{array}$ & Ampicillin & $50 \mathrm{mg} / \mathrm{kg} \mathrm{KG}$ i.v. \\
\hline \multirow[t]{3}{*}{$\begin{array}{l}\text { Penicillin- oder } \\
\text { Ampicillinallergie }\end{array}$} & Clindamycin & $\begin{array}{l}15 \mathrm{mg} / \mathrm{kg} \mathrm{KG} \\
\text { p.o. oder i.v. }\end{array}$ \\
\hline & Vancomycin & $20 \mathrm{mg} / \mathrm{kg} \mathrm{KG}$ i.v. \\
\hline & Teicoplanin & $10 \mathrm{mg} / \mathrm{kg} \mathrm{KG}$ i.v. \\
\hline
\end{tabular}

Anwendung von Amoxicillin oder Clindamycin dagegen $6 \mathrm{~h}$ nach dem Eingriff eine weitere Dosis (Amoxicillin $15 \mathrm{mg} / \mathrm{kg}$, Clindamycin $7,5 \mathrm{mg} / \mathrm{kg}$ ) erhalten.

Üblicherweise ist jeder der betroffenen Patienten im Besitz eines „Herzpasses für Kinder“, aus dem alle Informationen für den behandelnden Arzt zu entnehmen sind. Die aktuellen Empfehlungen sind der Website der Paul-EhrlichGesellschaft für Chemotherapie jederzeit aktuell zu entnehmen (www.p-e-g.org).

\subsection{Impfungen}

Um einer bakteriellen toxischen Infektion mit Clostridien vorzubeugen, ist laut STIKO (Ständigen Impfkommission am Robert-Koch-Institut 2011) eine Tetanus-Schutzimpfung empfohlen, die als Mehrfachimpfung verabreicht wird (z. B. in Kombination mit Diphtherie- bzw. Mumps- und Pertussisimpfung). Eine Tollwut-Schutzimpfung ist nur bei entsprechendem Verdacht erforderlich. Eine Pneumokokken- sowie eine Haemophilus-influenza-Impfung sollen bei vorgesehenen Milzteilresektionen bzw. Splenektomien erfolgen. Diese werden auch nach einer akuten Milzteiloperation (z. B. nach Unfall) empfohlen. Eine entsprechende Titerbestimmung ist auf jeden Fall erforderlich. Dagegen können Kinder mit Immundefizienz (Abschn. 4.4) auf Schutzimpfungen nicht adäquat reagieren, da Patienten mit B-Zell-Defekten in ihrer Fähigkeit, spezifische Antikörper nach Impfungen zu bilden, beeinträchtigt sind. Diese Kinder sollten mittels passiver Immunisierung geschützt werden, wobei aber die Effizienz einer solchen Impfung bezweifelt werden muss. Eine aktive Immunisierung kann dagegen fatale Folgen für solche Kinder haben. Davon ausgenommen sind Kinder mit einem selektiven IgA-Mangel, mit einem IgG-Subklassenmangel, mit Komplement- und Phagozytose-Defekten sowie mit einer Asplenie.

\section{Literatur}

Bjorkander J et al (2006) Prospective open-label study ofo pharmacokinetcs, efficacy and safety of a new $10 \%$ liquid intravenous immunoglobulin in patients with hypo- or agammaglobulinemia. Vox Sang 90:286-293

Brown EM, de Louvois J, Bayston R et al (1994) Antimicrobial prophylaxis in neurosurgery and after head injury. Lancet 344:1547-1551

Carbon RT, Lugauer S, Geitner U et al (1999) Reducing catheterassociated infections with silver-impregnated catheters in long-term therapy of children. Infection 27(Suppl 1):69-73

Duque-Estrada EO, Duarte MR, Rodrigues DM, Raphael MD (2003) Wound infections in pediatric surgery: a study of 575 patients in a university hospital. Pediatr Surg Int 19:436-438

Fätkenheuer G, Cornety O, Seifert H (2002) Clinical management of catheter-related infections. Clin Microbiol Infect 8:545-550

Graubner UB et al (2001) Vaccination. Klin Pädiatr 213(Suppl 1): A77-A83 
Hazer DB et al (2012) The efficacy of silver-embedded polypropylenegrafted polyethylene glycol-coated ventricular catheters on prevention of shunt catheter infection in rats. Childs Nerv Syst 28(6): 839-846

Huang EY et al (2011) Strategies for the prevention of central venous catheter infections: an American Pediatric Surgical Association Outcomes and Clinical Trial Committee systematic review. J Pediatr Surg Oct 46:2000-2011

Knerr V et al (2008) Die ESID-Online-Datenbank für primäre Immundefekte. Erste Analyse mit Blick auf Deutschland und Europa. Med Klin 103:620-627

Laux R, Wirtz S, Huggett S et al (2013) Bedeutung der Eltern bei der Besiedlung von Neonaten mit multiresistenten gramnegativen Erregern (MRGN). Z Geburtsh Neonatol 217:61-64

Mangram AJ et al (1999) Guidelines for prevention of surgical site infection. Infect Control Hosp Epidemiol 20:247-280

Mc Laughlin D, Friedmacher F, Puri P (2014) The impact of Clostridium difficile on paediatric surgical practice: a systematic review. Pediatr Surg Int 30:853-859

Mermel LA, Farr BM, Sheretz RJ (2001) Guidelines for the management of intravascular catheter-related infections. Clin Infect Dis $32: 1249-1272$
Naber CK, Al-Nawas B, Baumgartner H (2007) Prophylaxe der infektiösen Endokarditis. Kardiologe 1:243-250

Raymond J, Aujard Y, the European Study Group (2000) Nosocomial infections in pediatric patiants: a European, multicenter, prospective study. Infect Control Hosp Epidemiol 21:260-263

Reid MM (1994) Splenectomy, sepsis, immunisation and guidelines. Lancet 344:970-971

Robert-Koch-Institut (2011) Epidemiologische Bulletin. Mitteilungen der Ständigen Impfkommission am Robert-Koch-Institut. 01 Aug 2011, Nr. 30

Scholz H et al (2003) Infektionen bei Kindern und Jugendlichen, Handbuch der Dt. Gesellschaft für pädiatrische Infektiologie, 4. Aufl. Futuramed, München

Seifert H et al (2007) MIQ 3a: Blutkulturdiagnostik - Sepsis, Endokarditis, Katheterinfektionen (Teil I), Qualitätsstandards in der mikrobiologisch-infektiologischen Diagnostik, 2. Aufl. Urban \& Fischer/Elsevier, München

Smith-Slatas CL, Bourque M, Salazar C (2006) Clostridium septicum infections in children: a case report and review of the literature. Pediatrics 117:796-802

Wenzel RP (2007) Health care-associated infections: major issues in the early years of the 21st century. Clin Infect Dis 45:S85-S88 